\title{
Overlap and Repair of Turn- Taking System during Collaborative Oral Peer- Feedback in an EFL Writing Course
}

\author{
Fatimah Saadi Ali ${ }^{1}$ \\ ${ }^{1}$ English Language Teaching Department, Education Faculty, Tishk International University, Erbil, Iraq \\ Correspondence: Fatimah Saadi Ali, Tishk International University, Erbil, Iraq. \\ Email: Fatima.saadi@tiu.edu.iq
}

Doi: $10.23918 /$ ijsses.v8i2p128

\begin{abstract}
In the last decade, the researchers have suggested peer feedback in English as a foreign language (EFL) writing classroom. It is one of the professional activity tools to be provided in the EFL writing classroom, which makes the students involved more in the class. It makes classes learner-centred rather than teacher-centred. Therefore, many researches have been carried out about the usefulness, cognitive, and social benefits of peer feedback. This paper investigates overlap and repair, as two elements of the turn-taking system, that take place at the oral feedback writing session. The study also aimed to investigate how these elements happen and which of them is taken most. The qualitative approach was implemented in this study. The data was collected through audio recording. A group of freshmen students in an EFL writing course were participated. Their oral peer feedback session was recorded. The conversation analysis approach was used to analyse the interaction in the classroom. The study's result has shown that in the collaborative oral feedback session overlap and repair have taken place in the EFL writing classroom. It also revealed that it has a great role during the class session as well as an impact on those students who want to improve their writing skills. Overall, it has been recommended that collaborative feedback sessions should be investigated more in classroom conversation particularly in writing courses.
\end{abstract}

Key words: EFL Writing Course, Collaborative Oral Feedback, Overlap and Repair System

\section{Introduction}

Twenty-first-century instructors try to make their classrooms learner-centered. Receiving peer feedback, dividing assessment into smaller parts, and teaching the strategies of good writing are the aims of the trainers of writing course (Baker, as cited in Bean, 2011; Gibbs and Simpson, 2004; Grauerholz, 1999; Kamali, 1991; Kolb et al., 2013). In recent years, English as a Foreign Language (EFL) writing classes has become a place to practice students' work by giving them feedback. Baker (2016) suggested the benefits and quality improvements in a particular assignment, which is based on peer feedback. Nicol and Macfarlane-Dick (2006) pointed out that students can become independent writers. As an outcome of peer review or feedback. Their work is critically viewed from the perspective of the others that help them to improve themselves.

Received: April 17, 2021

Accepted: June 20, 2021

Ali, F.S. (2021). Overlap and Repair of Turn- Taking System during Collaborative Oral Peer-Feedback in an EFL Writing Course. International Journal of Social Sciences \& Educational Studies, 8(2), 128-134. 
Most of the recent studies on peer feedback have been concerned with paper-based corrective peer feedback. Either teacher-students or student-student interaction occurs in the class, although these two interactions have their impacts on the students' work. In this present study, the focus is on the collaborative oral feedback of students in the writing course. Lyster, Saito, and Sato (2012) explained in their research that student-to-student would do peer-interactions collaboratively oral feedback as they were seated in the class. Surely, while face-to-face feedback sessions are done orally, there will be some turn taking-system. In this study, to analyze the oral feedback session, two elements of the turn-taking system have selected to make them independent learners and supporting each other's ideas by turns. These are repairs and overlaps. These two elements are happening a lot in the oral peer feedback sessions on the writing tasks because each peer feedback takes its turn in this duration. These two elements will be in the feedback session.

Repair is defined as something that occurs in talk-in-interaction (Hellermann, 2009). Not clear, mishearing, incorrect information, and misspeaking are some cases that participants will do in the interaction. They lead to repairs either self-repair or other repairs (Hellermann, as cited in Schegloff, 1979; Schegloff, Jefferson, \& Sacks, 1977). Similar to that, Liebscher and DaileyO'Cain (2003) defined repair as "used to negotiate to mean and allows speakers to resolve the trouble in speaking, hearing, or understanding, output, and interaction, as well as modified input in classroom settings" (p. 375). It will happen when learners are working together to repair linguistically each other's paper [sic] to convey and understand each other's message. (Liebscher, and Dailey-O'Cain, as cited in Pica, 1996).

Hellermann (2009) has stated that he conversation analysis has declared the methods repair for the one who produces repair seem to be initiated as "self" or "other," which are shown as four sequences of repair. (A) Self-Initiated Self-Repair, (B) Other-Initiated Self-Repair, (C) OtherInitiated, Other-Repair (D) Self-Initiated Other-Repair. (p.114-116). On the other hand, overlap is one of the turn-taking mechanisms that pass between two speakers. As Wells and Macfarlane (1998) stated that "overlapping talk occurs which is not oriented to, either by the current speaker or by the incoming speaker, as to turn competitive". (p. 266).

This papers' aim is to investigate these three research questions:

1. When does the overlap take place during a peer feedback session in a writing class?

2. How does repair sequence as turn-taking system takes place during collaborative oral peer feedback session in classroom interaction?

3. Which type of repair are they going to take?

These kinds of interactions are different from mundane everyday conversations. It makes the student more familiar with their mistakes on the writing part. It also makes them be independent learners and critical thinkers. They will be aware of what is going on in the classroom too. It is worth it for those students who 
are not able to speak to the whole class especially, while peer feedback is a kind of great help to them to practice among students.

In conclusion, it has been cleared that collaborative oral feedback is one of the tools that make learners independent and critical thinkers as well. These two elements of the turn-taking system had a role in this interaction that will happen to them in the EFL writing class.

\section{Literature Review}

Nowadays, a lot of research studies have been conducted on the students' interaction during peer feedback. Each of them has analyzed the sequence of the turn-taking methods such as repair and overlap. This study has focused on the students' interaction. Wells and Macfarlane, (1998) have investigated the sources that make recipients don't have this display in talk-in-interaction. The study took place in a home of Halesowen town in England. The focal family had three members. An hour of the audio conversation was recording naturally. It was used to analyze the turns in talks. For example, the duration of the silence, location of the overlap onset, and using a sound scope. It was employed where the quality of recording permitted. Conversation Analysis was the method to analyze the conversation. The study aimed to figure out linguistic characteristics and where precisely overlapped being turned. The result was reported as those main points from these two studies. (a) differentiate of British English design to turn-competitive design concerning the pitch and volume, (b) differentiate in the design of overlap of Transition Relevance Place (TRP) as a space between TRP-projecting accent and another onset next turn, (c) hesitation did not display of pitches and volume's feature. (d) TRP-projection accent phonetically is different from the non-TRPprojection accent.

Collaborative repair and overlap methods that are happening simultaneously in the classroom interaction made researchers investigate these phenomena. Maroni, Gnisci, and Pontecorvo (2008) have carried out a study on the rhythm of classroom management. A primary state school was observed to collect the data during 15 hours in the 23 lessons. The participants were second, third, and fourth-grade students. The descriptive design was used to reveal those points. A. The transformation of children. B. interactive class. C. change in the turn-taking tactics. Overlap has changed teacher differs from children accordingly. Three main findings were found in this study: (a) changing of overlap according to the overlap, (b) differentiation of turn-taking strategies between students to students and teacher to students, and connectedness of the next speaker with pause duration and another speaker.

\section{Methods}

\subsection{Participants}

The study's participants were a group of students in an EFL writing course in the English Language Teaching Department of Near East University, North Cyprus. All participants of the group consented to be included in this study. The group members were chosen randomly, and they were four participants two males, and two females. Moreover, all participants' age was about 20-23 years old. All of them came from different nationalities and cultures. So, they have friended each other in this particular classroom. A2 level was the participants according to the Common European Framework of Reference for Languages 
(CEFR). One of the male participants had more experience giving and receiving peer feedback because he was retaking this course and had done similar activities in the previous class.

\subsection{Course Format and Data Collection}

Advanced Reading and Writing are the main courses of freshmen students in their second semester in the ELT department. The course was conducted for 14 weeks, and each week they were taught topics on how to write a research essay. In the last three weeks, how to give oral feedback was explained. They were familiar with the strategies of giving and receiving peer feedback. In the second week of peer feedback, the participants of the group were informed that the session would be recorded for two hours of the class, and all of them agreed. A mobile phone was used to audio-record the conversation during their feedback session. In this recording, only two minutes were taken to be transcribed and analysed.

\subsection{Data Analysis}

The conversational analysis approach was used to analyse the data as it is one of the approaches that fits to use to the data (Goodwin, 1981; ten Have, 1999; Schegloff, 2007; Schegloff et al., 1977; Wooffitt, 2005). The analysis concerns conversation structure and forms like turn-taking systems among the participants. Overlapping and the repairs were the study's purpose during feedback sessions occurring. The data was collected through audio recording. It has been transcribed and then analysed based on the approach.

\section{Findings and Discussion}

At this particular data, the general findings were that overlaps were happening by protecting each turns' idea on the referencing, during their feedback session. The classroom members were about 22 students. The class was divided into some groups each group was conducted with three to four participants. This particular group was four participants and were sitting opposite each other. They were giving feedback on Zaras' paper that she wrote on "the social media and its' effect on the language learning ". Before starting the part that has analyzed, they were reviewing the criteria of giving feedback on the referencing to make sure that they are ready to start the session. In this section, the detailed findings were explained by providing the sample of the extract.

The first line starts with Zara by "yeah." The function of this "yeah" is that she is ready as a receiver of the feedback to start the session. In lines 4 and 6, Zara agrees with what Daniel is saying. It is clear in lines $2,5,7$, and 11. Daniel is seen as a powerful figure in the group as he has taken this course before, and he knows the procedure. He leads the group can be seen in this part of the extract.

1 Zara: Yeah

2 Daniel: I think it doesn't matter because it is all of you if you if use to use because.

3 citation it a new $\mathrm{t}=$ hat

4 Zara =yeah 
5 Daniel: to support your ideas $=$

6 Zara: =yeah

7 Daniel: and interesting used [that

8 David: [whenever you want to support your ideas.

9 Daniel: yes

10 David: yes

11 Daniel: the right the right one [ is to support your ideas.

12 Rosie: [David, we write because ((inaudible)) yes.

The overlaps with Daniel are important in that his power position puts him in a more knowledgeable position compared to the others. Thus, under these circumstances, not many overlaps are expected. However, David in line 8 overlaps with Daniel to support him. His overlap reinforced Daniel's comment that the essay needs more about the supporting ideas. Even though he is overlapping to take the turn from him, David is acknowledging Daniel's position and makes sure that his support is not taken as a challenge. In lines 9 and 10, the function of these "yes" means they agreed with each other about the idea because they were talking about the same topic. In line 11, Daniel is still in the position. However, Rosie in line 12 overlaps with Daniel in a crowd situation trying to say something but it is not clear because of the noise in the class.

In a small part of the conversation, repair as one of the elements of the turn-taking system has happened. There were cases where the participant who speaks makes a mistake and tries to make the repair in a low tone of voice. There was one main repair that happened during the study. That is self-repair by asking questions to the other participants of the session. Self-repair is a sequence of repairs, whenever the turns are taking it. It means that they became an independent learner. As in lines 27 and 32, Rosie and Zara were taking it.

27 Rosie: ((inaudible)) if you ${ }^{\circ}$ wrote $^{\circ}$ write $\downarrow$ this one and paragraph $=(($ showing the part

28 to her roommate))

32 Zara: yeah because ah...Second resources I think uhh.. according to uhh... his name after that at the end of the citation uhh... ummm which. (0.5) how can we go second in- text citation $\downarrow$ second resources in text citation $\downarrow(0.3)$

In line 27, Rosie in a crowd situation asks, "if you wrote" instead of saying write makes a mistake. So directly, she repairs herself and changes the intonation of her voice into questioning. Line 32 presents that Zara starts with "yeah" with low voice and expressing some filler like "ah..., uhh, uhh, and umm" so that she can try to remember that it was "second resource" it appears with the last "umm" she could remember 
herself. After (0.5) pause duration, she says "second in text- citation" immediately she repairs herself by repairing "second resources in text citation" that is another self- repair has happened in the data.

\section{Conclusion}

In conclusion, it can be concluded that collaborative oral peer feedback played a role during the session in EFL writing class, and it enables the learner to be independent and critical thinkers. In addition, it was a huge chance for those students who wanted to improve their skills at writing. The main finding of the study, which analysed overlap and repair have happened a lot during the session among the participants. It is recommended that topic of the study be investigated more by researchers and taking place of overlap and repair in classroom conversation

\section{References}

Baker, K. M. (2016). Peer review as a strategy for improving students' writing process. Active Learning in Higher Education, 17(3), 179-192. Doi:10.1177/1469787416654794

Goodwin, C. (1981). Conversational organization: Interaction between speakers and hearers. New York, NY: Academic Press.

Hellermann, J. (2009). Looking for evidence of language learning in practices for repair: A case study of self-initiated self-repair by an adult learner of English. Scandinavian Journal of Educational Research, 53(2), 113-132. Doi:10.1080/00313830902757550

Liebscher,G., \& Dailey-O'Cain, J. (2003). Conversational repair as a role- defining mechanism in classroom interaction. The Modern Language Journal, 87(3), 375-390

Lyster, R., Saito, K., \& Sato, M. (2012).Oral corrective feedback in second language classrooms. Language Teaching, 46(01), 1-40. Doi:10.1017/s0261444812000365

Maroni, B., Gnisci, A., \& Pontecorvo, C. (2008). Turn-taking in classroom interactions: Overlapping, interruptions and pauses in primary school. European Journal of Psychology of Education, 23(1), 59-76. Doi:10.1007/bf03173140

Nicol, D., J., \& Macfarlane-Dick, D. (2006) Formative assessment and self-regulated learning: A model and seven principles of good feedback. Studies in Higher Education 31(2): 199- 218.

Sacks, H., Schegloff, E. A., \& Jefferson, G. (1974). A simplest systematics for the organization of turntaking for conversation. Language, 50(4), 696-735. Doi:10.2307/412243

Schegloff, E. A. (2007). Sequence organization in interaction: A primer in conversation analysis. Cambridge: Cambridge University Press.

Wells, B., \& Macfarlane, S. (1998). Prosody as an interactional resource: Turn-projection and overlap. Language and Speech, 41(3-4), 265-294. Doi:10.1177/002383099804100403

Wooffitt, R. (2005). Conversation analysis and discourse analysis: A comparative and critical introduction. London, England: Sage 


\section{Appendix}

Transcription Conventions

Bold and underline indicate stress.

\begin{tabular}{|c|l|c|}
\hline [ & Overlap talk \\
\hline$\downarrow$ & & fall voice \\
\hline$\uparrow$ & & rising voice \\
\hline( & )$)$ & inaudible word or phrase with bold \\
\hline( & \\
\hline
\end{tabular}

(0.1) Length of silence in tenth of second [sic] speaker mistake

(()) words/phrases inserted to identify the addressee.

${ }^{\circ}$ wrote ${ }^{\circ}$ the talk between them is markedly softer than the talk around it. Bold indicates repairs. 